\title{
Article \\ Joint Selection of Influential Users and Locations under Target Region in Location-Based Social Networks
}

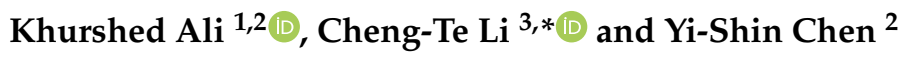 \\ 1 TIGP-SNHCC, Institute of Information Science, Academia Sinica, Taipei 115, Taiwan; \\ khurshed@iis.sinica.edu.tw \\ 2 Institute of Systems and Applications, National Tsing Hua University, Hsinchu 300044, Taiwan; \\ yishin@cs.nthu.edu.tw \\ 3 Institute of Data Science, National Cheng Kung University (NCKU), Tainan 701, Taiwan \\ * Correspondence: chengte@mail.ncku.edu.tw
}

Citation: Ali, K.; Li, C.-T.; Chen, Y.-S Joint Selection of Influential Users and Locations under Target Region in Location-Based Social Networks. Sensors 2021, 21, 709. https://doi. org/10.3390/s21030709

Received: 17 December 2020

Accepted: 15 January 2021

Published: 21 January 2021

Publisher's Note: MDPI stays neutral with regard to jurisdictional clai$\mathrm{ms}$ in published maps and institutional affiliations.

Copyright: (C) 2021 by the authors. Licensee MDPI, Basel, Switzerland. This article is an open access article distributed under the terms and conditions of the Creative Commons Attribution (CC BY) license (https:// creativecommons.org/licenses/by/ $4.0 /)$.

\begin{abstract}
Influence Maximization problem, selection of a set of users in a social network to maximize the influence spread, has received ample research attention in the social network analysis domain due to its practical applications. Although the problem has been extensively studied, existing works have neglected the location's popularity and importance along with influential users for product promotion at a particular region in Location-based Social Networks. Real-world marketing companies are more interested in finding suitable locations and influential users in a city to promote their product and attract as many users as possible. In this work, we study the joint selection of influential users and locations within a target region from two complementary perspectives; general and specific location type selection perspectives. The first is to find influential users and locations at a specified region irrespective of location type or category. The second perspective is to recommend locations matching location preference in addition to the target region for product promotion. To address general and specific location recommendations and influential users, we propose heuristic-based methods that effectively find influential users and locations for product promotion. Our experimental results show that it is not always an optimal choice to recommend locations with the highest popularity values, such as ratings, check-ins, and so, which may not be a true indicator of location popularity to be considered for marketing. Our results show that not only influential users are helpful for product promotion, but suitable influential locations can also assist in promoting products in the target region.
\end{abstract}

Keywords: recommendation system; influence maximization; social network analysis; viral marketing; location-based social networks

\section{Introduction}

Influence Maximization (IM) problem, which finds $k$ users from a social network to activate a maximum number of users, is a widely studied research topic [1-6]. Domingos and Richardson [7,8] are the first to study the problem as an algorithmic problem and modeled the problem using Markov random fields. The IM problem is formulated as a discrete stochastic optimization problem by Kempe et al. [1] and used two models, i.e., Independent Cascade (IC) model and Linear Threshold model, to describe the influence propagation over social networks.

In addition, researchers have analyzed the location-based social networks (LBSN) [9,10]. LBSN provides a platform to users to "check-in" and share the location information with their online friends. Considering the popularity of LBSNs, the IM problem has also been studied on location-based social networks (LBSN) [11-14]. Li et al. [11] have formulated the location-aware influence maximization problem. Given a query region and users' locations, the problem is to find influential users that could maximize the influence spread in the query region. Bouros et al. [12] have tried to solve a similar problem as proposed in [11] of finding influential users at query region, but he ranked the influential regional users 
rather than finding a group of users which collectively maximize the influence spread in the query region. Finally, a very recent work by Wang et al. [14] on IM with similar context to our work tries to find regionally influential users considering the distance between seed set and influenced users in a target region. However, none of the previous work has considered a selection of influential users and locations simultaneously for product promotion. Evidently, in a real-world marketing scenario, business companies are as keen on finding suitable venues as selecting a set of key users that could provide maximum profit.

Due to the popularity of smartphones and location-based social networks (LBSNs), users can check-in at locations or venues and share their check-in information with their friends and family. This free word-of-mouth marketing phenomenon facilitates companies to promote their products by exploring users' check-in sharing history to attract more users to visit. However, location popularity values, such as check-ins and ratings, are not always available in each location-based social network, except that we are provided only the user social network and set of locations. Thus, it is challenging to find influential users and locations in the target region simultaneously. Further, it is also not sure that the selected locations based on popularity values are always good choices for product promotion. We discuss this issue using the example in Figure 1. In this example, we have selected the top-20 locations across San Francisco from Gwalla dataset based on the number of users and check-in frequency. It can be observed that some locations have the highest check-ins, but these are visited by only few users (marked in red font). So It can be misleading to consider location popularity values only for product promotion. To address this problem, we find influential users and locations which can provide a solid platform for product promotion irrespective of location popularity values.

\begin{tabular}{|c|c|}
\hline \#checkins & \#users \\
\hline 171341059 boof964a520850b1fe3 & 682 \\
\hline 919 4bd2177d046076b055357371 & 1409 \\
\hline 364 4a5824e5f964a5206db71fe3 & 210 \\
\hline 323 4ab595e1f964a520877520e3 & 167 \\
\hline 44049 d01698f964a520fd5a1fe3 & 161 \\
\hline $3334 \mathrm{a} 71 \mathrm{e} 4 \mathrm{cff} 964 \mathrm{a} 520 \mathrm{ccd} 91 \mathrm{fe} 3$ & 133 \\
\hline $17149 c c 413 d f 964 a 5205 a 591 f e 3$ & 123 \\
\hline $16943067280 f 964 a 52023271 \mathrm{fe} 3$ & 119 \\
\hline $12842 \mathrm{cc} 7080 \mathrm{f} 964 \mathrm{a} 520 \mathrm{eg} 251 \mathrm{fe} 3$ & 104 \\
\hline 101 40c3b000f964a520e7001fe3 & 82 \\
\hline 106 409ad180f964a520eef21ee3 & 82 \\
\hline $12349 \mathrm{fdea} 19 \mathrm{fg} 64 \mathrm{a} 5204 \mathrm{a} 6 \mathrm{f} 1 \mathrm{fe} 3$ & 80 \\
\hline $10040943 a 00 f 964 a 520$ e5f21ee3 & 78 \\
\hline 108452 b81ddf964a520393b1fe3 & 74 \\
\hline $82409 d 7480 f 964 a 520 f 2 f 21 e e 3$ & 73 \\
\hline $1024249 e c 00 f 964 a 5208 f 201 \mathrm{fe} 3$ & 73 \\
\hline $8940 \mathrm{bbc} 700 \mathrm{f} 964 \mathrm{a} 520 \mathrm{~b} 1001 \mathrm{fe} 3$ & 73 \\
\hline 934 bb 9 dbc $353649 c 74364 \mathrm{e} 48 \mathrm{fb}$ & 72 \\
\hline $107444 \mathrm{df} 333 \mathrm{f} 964 \mathrm{a} 5208 \mathrm{~d} 321 \mathrm{fe} 3$ & 72 \\
\hline $893 f d 66200 f 964 a 52041$ ee1ee3 & 70 \\
\hline
\end{tabular}

With highest user frequency

\begin{tabular}{|c|c|}
\hline \#checkins & \#users \\
\hline 1713 41059b00f964a520850b1fe3 & 3682 \\
\hline 919 4bd2177d046076b055357371 & 1409 \\
\hline 44049 d01698f964a520fd5a1fe3 & 161 \\
\hline $3644 a 5824$ e5f964a5206db71fe3 & 210 \\
\hline $3334 a 71$ e4cffg64a520ccd91fe3 & 133 \\
\hline 323 4ab595e1f964a520877520e3 & 167 \\
\hline $19349 f 7968 \mathrm{df} 964 \mathrm{a} 520 \mathrm{bf} 6 \mathrm{c} 1 \mathrm{fe} 3$ & 36 \\
\hline 175 4acfadeef964a52049d520e3 & 11 \\
\hline $17149 c c 413 d f 964 a 5205 a 591 f e 3$ & 123 \\
\hline $16943067280 f 964 a 52023271 \mathrm{fe} 3$ & 119 \\
\hline $16140 \mathrm{ca} 4780 \mathrm{f} 964 \mathrm{a} 52008011 \mathrm{fe} 3$ & 47 \\
\hline $15340 \mathrm{~b} 7 \mathrm{~d} 280 \mathrm{fg} 64 \mathrm{a} 52093001 \mathrm{fe} 3$ & 61 \\
\hline $1504 c 2 f 9 c 1 d 6 f 1$ fef3b2c94eb3d & 1 \\
\hline $15042853 f 80 f 964 a 5200 c 231 \mathrm{fe} 3$ & 49 \\
\hline 146 4ab81d6ff964a5203d7c20e3 & 7 \\
\hline $143455 f 77 a b f 964 a 520903 d 1 f e 3$ & 62 \\
\hline 130 4a4aa5a7f964a5200bac1fe3 & 2 \\
\hline $12842 \mathrm{cc} 7080 f 964 a 520 \mathrm{e} 9251 \mathrm{fe} 3$ & 104 \\
\hline $12543598100 f 964 a 520 f 7281 \mathrm{fe} 3$ & 7 \\
\hline 123 49fdea19f964a5204a6f1fe3 & 80 \\
\hline
\end{tabular}

With highest checkin frequency

Figure 1. Top-20 locations across San Francisco from Gwalla Dataset.

In short, we answer the following research questions:

1. Is the location check-in frequency always an optimal choice for product promotion in LBSN?

2. How much percent influenced users belong to the query region, and should we consider influenced users out of the region for product promotion?

3. What users and locations to suggest for product promotion matching query region and query topic without considering location popularity information?

To address the aforementioned challenge, we propose two main approaches that select influential users and locations irrespective of location popularity values at the target region. The first approach referred as Region-Aware Influential users and locations selection (IUL), 
consists of algorithms which select influential users and locations within a target region $R$ for product promotion. In-Region (IR Selection) algorithm selects $k$ users and $m$ locations which are under target region $R$. Region-Free (RF Selection) algorithm considers users who are out of region but connected with users inside target region for finding $k$ influential users and $m$ influential locations. Greedy-In Region (G-IR Selection) algorithm selects $k$-influential users and $m$-influential locations by considering check-in frequency. It is worth noting that we are not provided the boundary area of the target region except in some LBSNs such as Foursquare and so. So we have extracted all the locations which are under a certain distance from the target region. Initially, we find $k$ influential users under the target query region and then extract top- $m$ locations, visited by $k$ influential users, with highest overall users frequency.

The second approach, named topic-aware influential users and locations selection (t-IUL), selects influential users and locations matching the query region and query topic. The algorithms proposed for this approach are similar to the ones proposed in the first approach but with the additional constraint of a specific type of location selection. To sum it all, we make the following contributions.

- We formally define the influential seed and location selection problem over locationbased social networks.

- We formally define the topic-aware influential seed and location selection problem over location-based social networks.

- We propose heuristic-based algorithms for influential users and location selection simultaneously for product promotion.

The rest of the paper is organized as follows. Section 2 reviews the related work. Section 3 describes the problem of identifying influential users and locations at a query region in LBSNs. We discuss the proposed frameworks in Section 4. Section 5 show our experimental results, followed by discussion and conclusion in Section 6.

\section{Literature Review}

\subsection{Influence Maximization in Social Network}

The influence maximization (IM) problem has been extensively studied in literature $[1-5,7,8,11,15]$. The IM problem was proposed by Domingos et al. in their seminal work [7]. The authors proposed probabilistic methods to address the IM problem. Kempe et al. [1] utilized two models, Independent cascade (IC) and Linear threshold (LT), to solve the IM problem. Futher, he proved the monotonic and the submodular property of the influence spread function and the problem's hardness (i.e. NP-hard). As the problem is NP-hard, the authors proposed the greedy algorithms with the approximation ratio of $\left(1-\frac{1}{e}\right)$. Further, research is carried out to improve the efficiency and scalability of the greedy algorithm while maintaining the approximation ratio. Leskovec et al. [15] proposed a lazy forward approach which achieves 700 times better efficiency performance than the algorithm proposed in [1]. Chen et al. [2] proved that it is \#P-hard to compute the influence spread. Thus, many algorithms rely on heuristic strategies to enhance performance. Chen et al. [2] utilized the degree discount heuristic to compute the influence spread for influence maximization problem. Recently, a near-linear time approach is proposed by Borgs et al. [4], which utilizes the Monte-Carlo Simulations to compute the influence spread. Further, Ohsaka et al. [5] proposed a pruning technique for reducing Monte-Carlo simulations time complexity to achieve better results with theoretical guarantees.

\subsection{Influence Maximization in Geo-Social Network}

With the increase in the use of location-enabled devices, the location factor carries an important role in social network analysis. Zhu et al. [13] consider a geo-social network where each user is associated with multiple check-ins. The authors proposed algorithms to compute the propagation probability distribution based on user check-in history in a promoted location. The works mostly related to our work are [11,12,14]. Li et al. [11] selected a seed set which globally maximizes the influence spread in a query region. Bouros et al. [12] 
used the Dijkstra algorithm to compute the regional influence of all users at a specific region and rank the influential users accordingly. Wang et al. [14], have considered a distance between seed set and promoted location for assigning edge weight. They have assigned different weights to users based on the distance between a user and the promoted location.

All of the aforementioned works have considered the location popularity and tried to find the seed set, which can globally maximize the influence spread. Our work mostly resembles to the work proposed by Bouros et al. [12], but we recommend influential locations along with an influential seed set for product promotion at a target region in location-based social networks.

\subsection{Location Recommendation in LBSNs}

Researchers have investigated the location-based social networks for analysis as well $[9,10,16]$. Authors in [9] analyzed the LBSN, its properties, and possible issues related to it. While, Ahmed et al. [16] discussed the importance of different centrality measures for identifying a node in geo-based social networks. Besides, Venue or location recommendation matching user query in LBSN has been well studied in literature [17-22]. Ye et al. [21] used collaborative filtering recommendation system by developing friend-based collaborative filtering system to recommend locations to users. Levandoski et al. [18] used the user ratings to locations for venue recommendation. Yuan et al. [17] proposed a time-aware POI recommendation problem, which suggests a list of venues for a user to visit at a given time. A recent work by Zhang et al. [23] recommends locations based on users preferences learning from the community-contributed data. The work done by Chang et al. [23] is similar to our $t$-IUL problem, but we recommend venues to merchants which satisfy merchant's query topic and target region by considering influential users in mind.

\section{Problem Formulation}

$\operatorname{A} \operatorname{LBSN}\langle G, L\rangle$ consists of a social network $G=(U, E)$, where $U$ is the set of users, $E$ is the set of edges and the set $L$ represents the set of locations with users check-in history as $\left\{\left(u, l_{1}, t\right)\right\}$, where $\left(u, l_{1}, t\right)$ represents a check-in record of user $u$ at location $l_{1}$ at time $t$, and $l_{1} \in L$. A location $l_{1}$ consists of latitude, longitude and location id. For $t-I U L$ problem, we assume that each location has category type which defines the type of location.

Definition 1. Influential Users: Influential users are a set of key users selected by companies that can propagate companies product information to a large number of users in a social network.

Definition 2. Influential Locations: Influential locations are locations that help companies spread their product information to a large volume of users even without investing in seed users set.

Now, we formally define the joint selection of influential users and locations problem (IUL) as following.

Definition 3. IUL Problem: Given a LBSN $\langle G, L\rangle$, a query region $R$, constants $k$ and $m$, the IUL problem is to select a set of users $S$, where $S \in U,|S|=k$, and set of locations $V$, where $V \in$ $L,|V|=m$, which both can help promote products at target region $R$ and attract as many users as possible to adopt products.

We illustrate the importance of the joint selection of influential users and locations in the following example.

Example 1. Figure 2 shows locations with users check-in frequency. We suppose all locations lie under a target query region. Under this query region, top-2 locations $(m=2)$ would be locations $A$ and location $C$ having the most users and highest check-ins, respectively. Although top-2 locations are locations $A$ and $C$, in fact, we do not know the actual visited users and check-ins 
at the target region. So, it is not easy to find influential users and locations under a query region for product promotion.

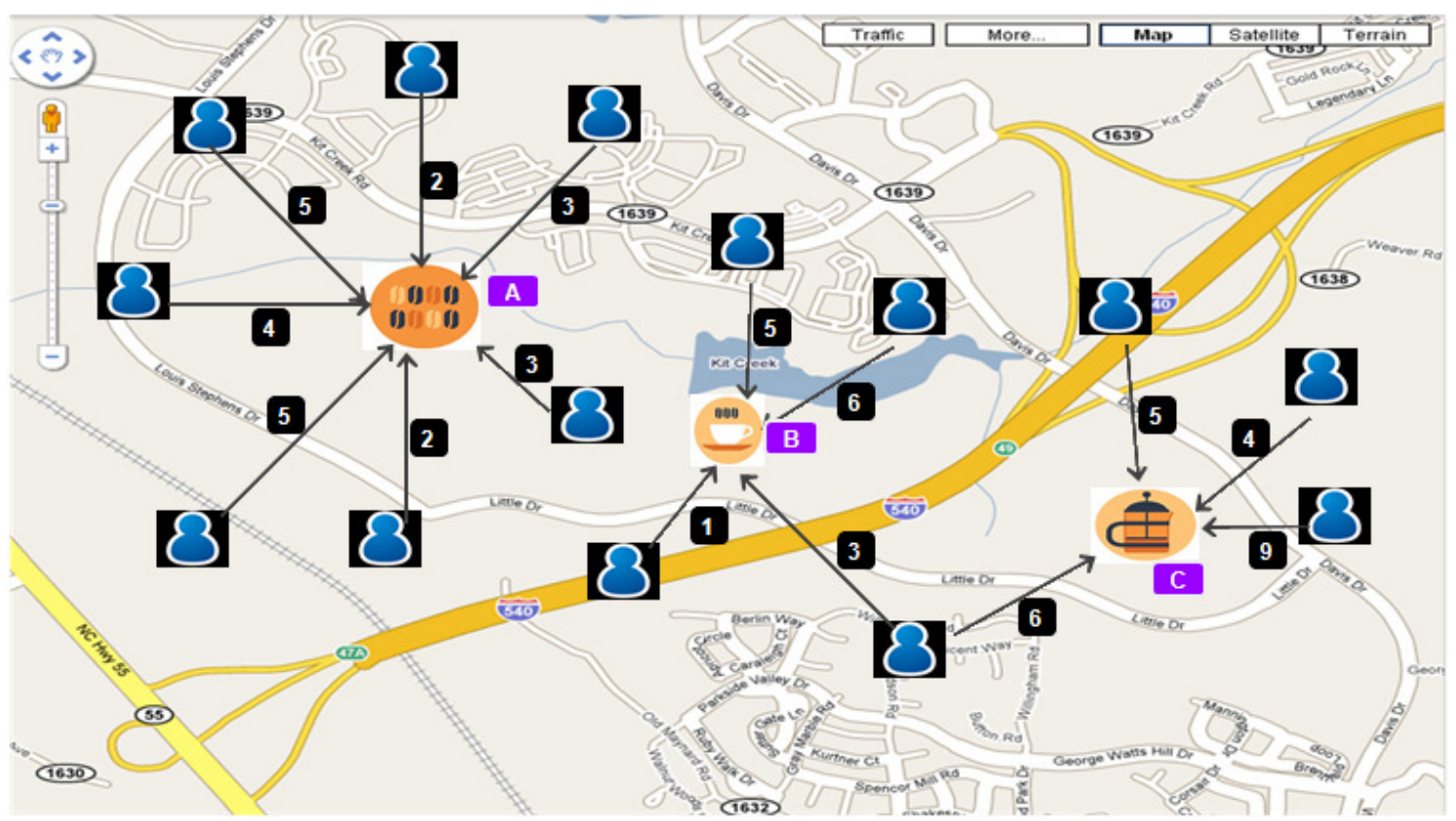

Figure 2. Example Figure.

Next, we define the Topic-Aware selection of influential users and locations ( $t$-IUL) problem as follows.

Definition 4. $t$-IUL Problem: Given a LBSN $\langle G, L\rangle$, a target region $R, a$ constant $m$ and a location category $C$, the $t$-IUL Problem is to select a set of seeds $S$, where $S \in U,|S|=k$, and set of locations $V$, where $V \in L,|V|=m$, matching a query category $C$, which can help companies to promote product at target region $R$ and spread their product information to large number of users in a target region.

\section{Methodology}

Section 4.1 discusses the approaches for solving IUL problem followed by strategies for solving t-IUL problem in Section 4.2.

\subsection{IUL Approaches}

Given a LBSN $\langle G, L\rangle$, a target region $R$, constants $k$ and $m$, the problem is to select $k$ influential users and $m$ influential locations that can help to promote products. Initially, we extract all the locations which are within target query region $R$ followed by social sub-graph construction of users who have checked-in at extracted locations. Then influential $k$-seeds are found by using degree discount proposed by Chen et al. [2]. It should be noted that we can find influential $k$-seeds using other approaches too, such as proposed by Ohsaka et al. [5], and Borgs et al. [4]. Finally, we select all locations visited by $k$-seeds and select top- $m$ locations from these locations based on highest number of users visiting those locations.

We propose three heuristic-based methods, In-Region Selection IR Selection, Region-Free Selection RF Selection and Greedy In-Region Selection G-IR, to address the IUL problem.

IR Selection. In this method, we consider all those users who have checked-in at least once within a query region boundary for influential seeds as well as the targeted users who can be influenced by product promotion. This approach is suitable when the marketing 
company wants to target the users who have frequently visited the places within a query region boundary and have a social connection with each other.

The Algorithm 1 selects the influential users and locations within a target query region $R$ using the IR Selection approach.

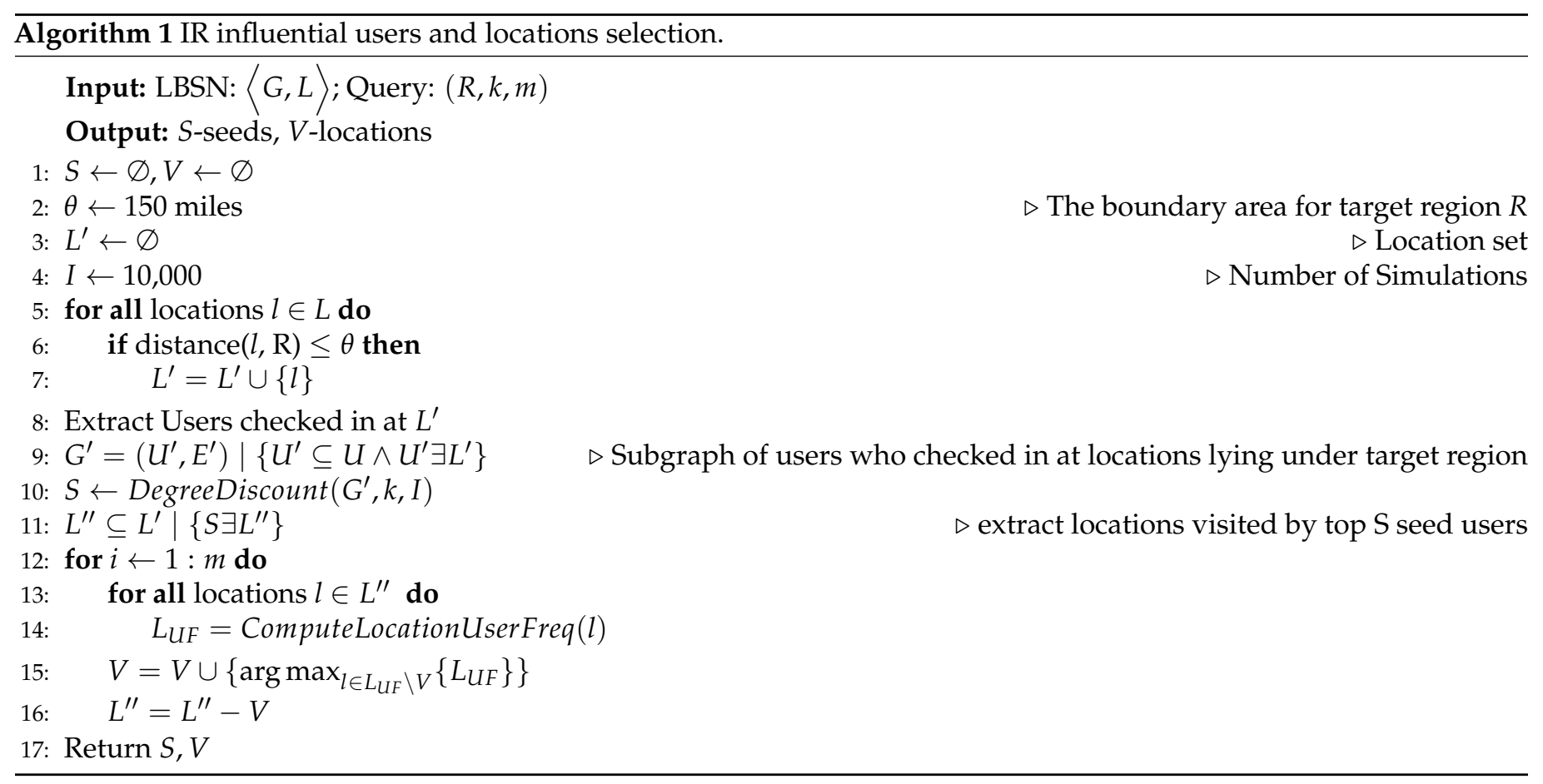

In this Algorithm 1, we consider users within a target query region $R$. Line 2 computes the boundary area of a target region $R$. Here, we assume the boundary area of each target city as 150 miles. It means we extract all locations which lie under 150 miles of a target region/city (i.e., New York). $L^{\prime}$ and $I$ represent the locations within target region and number of simulations to run for influence at line 3 and 5 respectively. We extract all the locations which lie under target region boundary from lines 5 to 7 . After getting all the locations within a target region, a social sub-graph, $G^{\prime}$, is constructed of users who checked-in at those locations, i.e., line 8 selects users who checked in at locations $L^{\prime}$ which lie under a target query region. We extract top-k seeds at line 9 using the degree discount method. Line 10 of this algorithm extracts the locations, $L^{\prime \prime}$, visited by top- $k$ influential seeds. From line 14 to 15 , we compute the location's user visiting frequency, $L^{\prime \prime}$, and each location is stored along with the number of users (not only top-k seeds but all users) who checked-in at these locations. Top- $m$ locations are selected by extracting locations with highest user frequency from $L^{\prime \prime}$ at line 17 to 19 .

RF Selection: In the region-free selection approach, users who have checked-in at least once at query region $R$ boundary are selected along with the inclusion of their children who can be within target region or out of target region boundary. As we are not provided the user's home location, so, we consider users having social links with users inside the target region boundary. This approach is beneficial when we do not know the actual home location of users but can target them if they have any social ties with users inside the target region.

The Algorithm 2 discusses the selection of influential users and locations using the RF selection approach. In this algorithm, line 1 extracts all those users who have checked-in at least once within a target query region, $R$, boundary first. Next, we add the child nodes of users who were selected earlier (at line 1) from lines 3 to 6 . Further, we select the $k$-influential users and $m$ influential locations from line 7 to line 13 in the same way as discussed in Algorithm 1. 


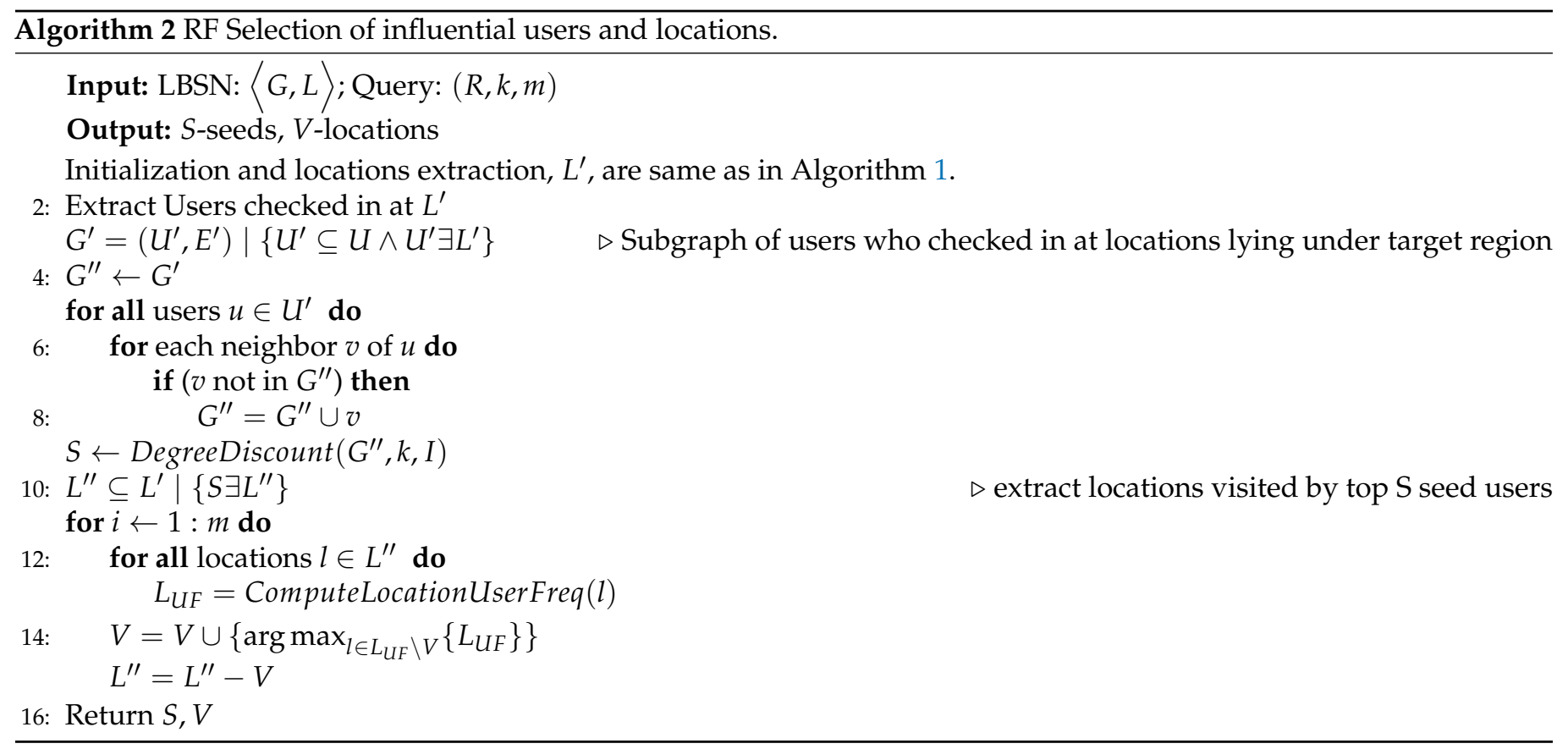

G-IR Selection. The Greedy in-region selection approach selects top- $k$ influential users and $m$-locations with highest number of check-ins. It is assumed that locations and users with the highest number of check-ins are suitable for product promotion in the target region and can spread product information to many users in a region.

The Algorithm 3 discusses the greedy approach for finding top- $k$ influential users and $m$-influential locations. Line 1 extracts the users who have checked-in at target query region $R$. From lines 2 to 6 , we select $k$-influential users considering the user's highest number of check-ins. Next, we select top $m$-influential locations with the most number of visits, from line 7 to 13 .

Each proposed heuristic-based methods carries pros and cons with it. For instance, the IR-Selection approach focuses only on users who are within the target region boundary but lacks users consideration who are indirectly inside the target region boundary. The RFSelection approach considers both users, i.e., users directly inside region boundary and indirectly within region boundary, for product promotion.

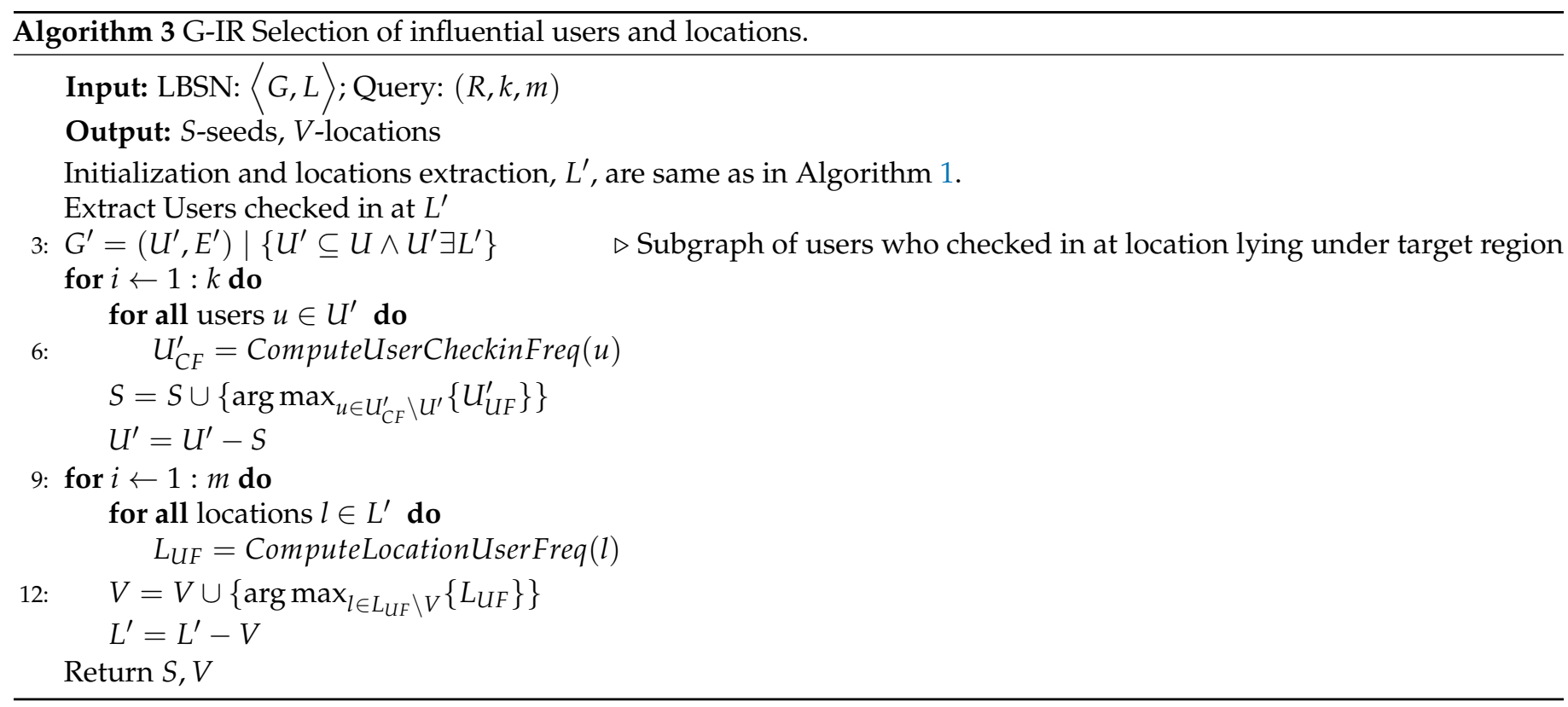


Further, the effectiveness of each approach, i.e., In-Region Selection (IR Selection), Region-Free Selection (RF Selection), and Greedy In-Region Selection (G-IR), is discussed in Section 5.

\section{2. -IUL Approaches}

$t$-IUL problem is to select influential users and locations attarget query region matching a specific query topic.

We propose two heuristic-based approaches to solve t-IUL problem; topic-aware RegionFree Selection (t-RF selection) and topic-aware Greedy In-Region Selection (t-GIR selection).

t-RF Selection. Topic-Aware Region free selection approach is similar to the RF-Selection approach of IUL problem with one more constraint in location selection. Here, we consider locations lying within a target query region boundary and matching the query topic that can be the merchant's own interest or preference for product promotion.

The Algorithm 4 illustrates our topic-aware region free selection approach. We compare the location type/category with a given query type. We have designed this algorithm considering Foursquare dataset, which have location city and category information. It can be extended to any other LBSNs dataset, provided that every location has a category or topic field. Lines 2 to 5 of this algorithm extracts those locations which match the target query region and query topic. The remaining part of the algorithm is the same as the RF-Selection algorithm and discussed earlier in IUL approach.

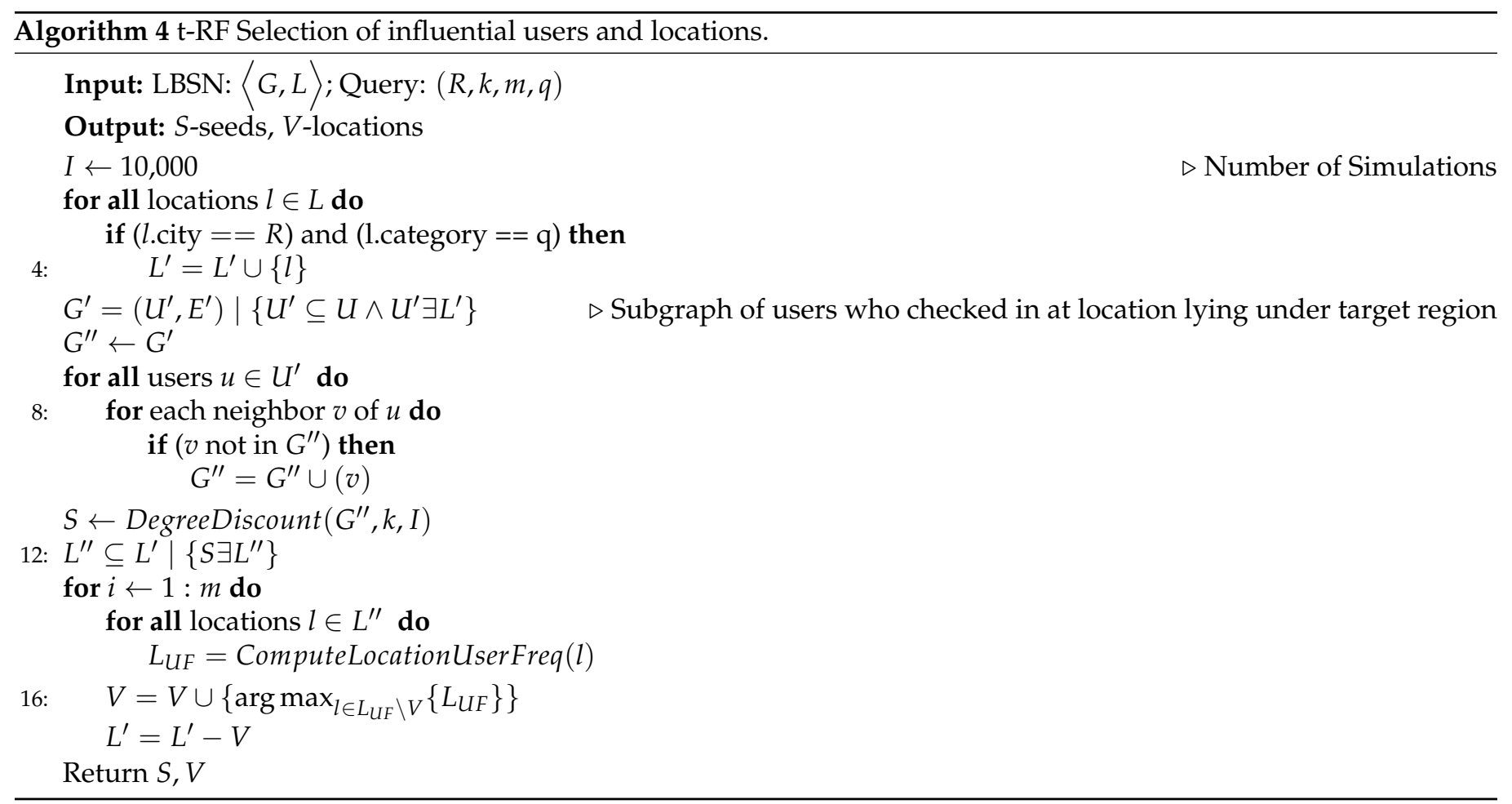

t-GIR Selection. Topic-Aware Greedy In-Region approach resembles the G-IR Selection approach of IUL problem with additional constraint in location selection. Here, we consider locations belonging to the target query region and matching the merchant's preferred query topic for product promotion.

The Algorithm 5 presents the greedy approach for selecting influential users and locations matching user query topic. It is worth noting that these both Algorithms 4 and 5, can easily be used to find $m$-influential locations to recommend to users for visiting (from consumer's perspective). 


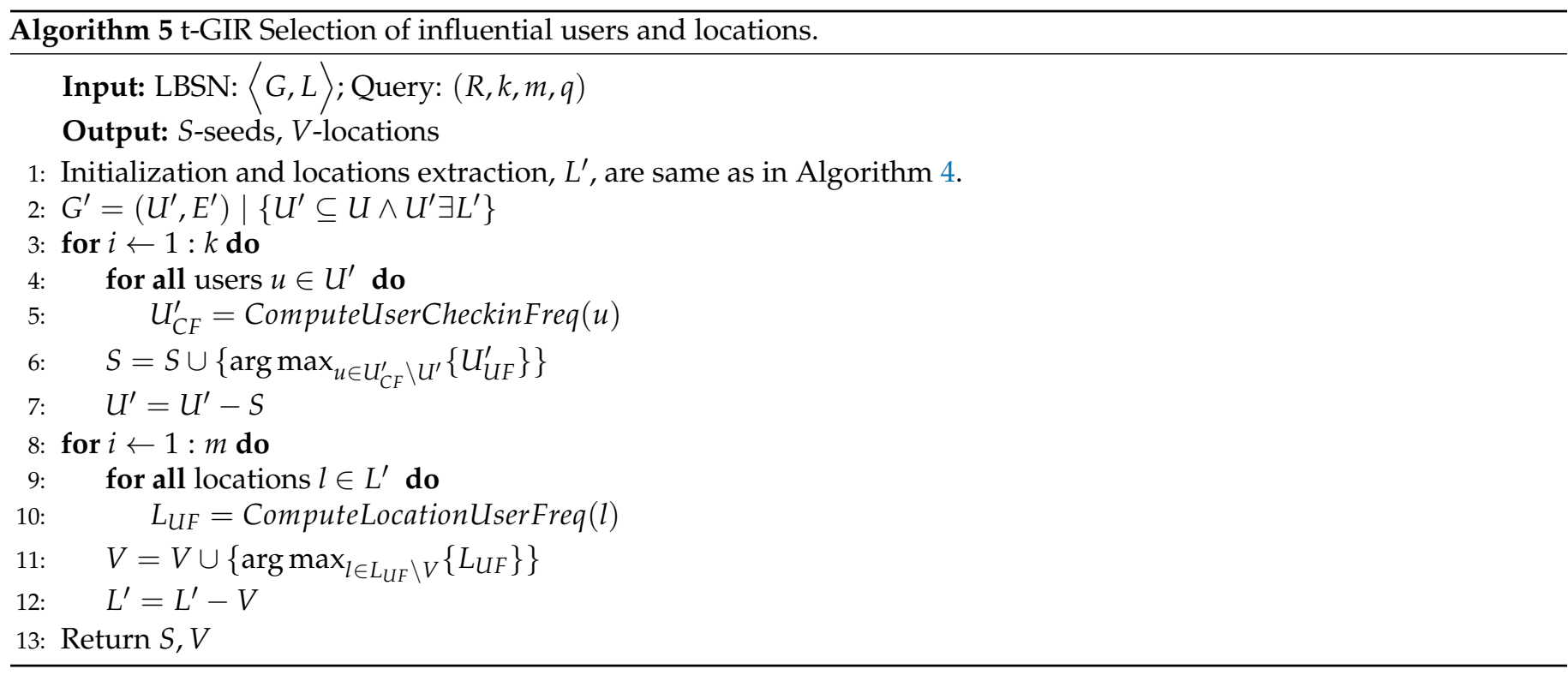

\subsection{Time Complexity}

Time Complexity: Let $L$ denotes the total number of locations in LBSN, $L^{\prime}$ represents number of locations in target region, $L^{\prime \prime}$ number of locations visited by influential seeds. And $n$ represents the total number of users, $n^{\prime}$ - number of users checked-in at target region, $m$-number of edges.

Algorithms 1 and 2 use the degree discount [2] heuristic-based method. As mentioned by Chen et al. [2], the running time of it is $O\left(k \log n^{\prime}+m\right)$. So the running time of Algorithm 1 is $O\left(L+n L^{\prime}+k \log n+m\right)$ and the running time of Algorithm 2 is $O\left(L+n L^{\prime}+n n^{\prime}+\right.$ $k \log n+m)$. Though the Algorithm 1 is faster than the Algorithm 2 but it lacks user selection who have directly social connection with users inside target region and could be useful for marketing the company products.

The running time of Algorithm 3 is $O\left(L+n L^{\prime}+k n^{\prime}+m L^{\prime}\right)$. The greedy In-Region selection approach is faster than the other two approaches. However, it does not provide users and locations which are beneficial for product promotion as discussed in Section 5 .

The time complexity of Algorithms 4 and 5 is the same as the running time complexity of Algorithm 2 and 3, respectively.

Further, we discuss the effectiveness of our proposed algorithms in results Section 5.

\section{Experiments}

We conducted experiments of our proposed algorithms on three real-world locationbased social networks, as discussed in Table 1. The objective is to find which algorithms can provide better and effective results for product promotion under certain constraints, such as within target region $R$ and location type $q$. Besides, we analyze the recommended users and locations for product promotions about how and why these results would be helpful for product promotion in a target region.

\subsection{Experimental Setup}

Datasets. We used three real world datasets Gowolla, Brighkite, Foursquare. Gowalla and Brighkite data sets are publicly available at stanford website [24]. While the Foursquare date set (http:/ / net.pku.edu.cn/daim/yinhongzhi/index.html), used by Wang et al. in [25], was downloaded from his website (publicly made available for download). The three datasets are directed graphs and details of each data set is discussed in Table 1. 
Table 1. Datasets.

\begin{tabular}{cccc}
\hline Datasets & \#Vertices & \#Edges & \#Check-ins \\
\hline Gowalla & 196,591 & 950,327 & $6.4 \mathrm{M}$ \\
\hline Brighkite & 58,228 & 214,078 & $4.49 \mathrm{M}$ \\
\hline Foursquare & 4163 & 32,512 & 483,813 \\
\hline
\end{tabular}

Comparison Methods. We compare our heuristic-based algorithms (Algorithms 1, 2 and 4 ) with greedy approaches (Algorithms 3 and 5). For the heuristic methods, we used the degree discount approach [2] to find the seed set and compute the influence spread. Finding the influential users and their influence spread can easily be done by other stateof-the-art approaches, such as [4-6].

Target Query Region. We have selected the big modern cities of the U.S., such as New York, Chicago, San Francisco, Washington, Seattle, Las Angeles, as our target query region and computed the influential users and locations selection within a specified target region. For each city, we extracted its geo-coordinates, i.e., latitude and longitude, and then selected all those check-in venues which lie under the target region. Locations within the target region are extracted using Vincenty distance formula. Since the Foursquare dataset contains city information, so we extracted all locations matching the query region.

Propagation Probability. We randomly selected each edge's propagation probability from $\{0.1,0.01,0.5,0.001\}$ as mentioned in [3].

Parameters. We measure the effectiveness of the proposed approaches for reporting region-aware influence spread, comparing selected influential users and locations. The seed set size $k$ varies from 10 to 50 and set $\mathrm{m}$-locations as 20 .

The influence spread of heuristic approaches is obtained by running 10,000 simulations for each set and took the average of the influence spread that matches the settings in [3]. All the proposed approaches are implemented in Python. Experiments are conducted on a PC with Intel Core(TM) i7 3.6 GHz and 8 GB memory using Windows 10.

\subsection{Experimental Results}

We evaluate the effectiveness of heuristic-based algorithms with greedy-based algorithms proposed in IUL and $t$-IUL approaches by varying seed set size $k$. We discuss the experimental results of IUL and $t-I U L$ approaches in Sections 5.2.1 and 5.2.2 respectively.

\subsubsection{IUL Approaches Result Summary}

First, we discuss the influence spread result achieved by IR, RF, and G-IR selection algorithms. As all the algorithms showed similar behavior on the Brightkite dataset as they performed on the Gowalla dataset, so we have excluded the Brightkite analysis result summary here.

Figure 3 shows the influence spread within or out of target cities where locations lie under 150 miles of the target city on the Gowalla dataset. It can be seen that there is a huge difference in influence spread within and out of the target city achieved by RF, IR, and G-IR algorithms. Like in Figure 3a, when $k=20$, the number of influenced users achieved by G-IR, IR, and RF algorithms are around 100, 180, and 650, respectively. There is a significant percentage difference in influence spread within and out of the target region. Similar difference is observed in other target cities as shown in Figure 3b-d. We conducted the same experiment on other cities of U.S., such as Seattle, California, Las Angles, and Los Vegas, and found the same influence spread difference in these target cities as well. 


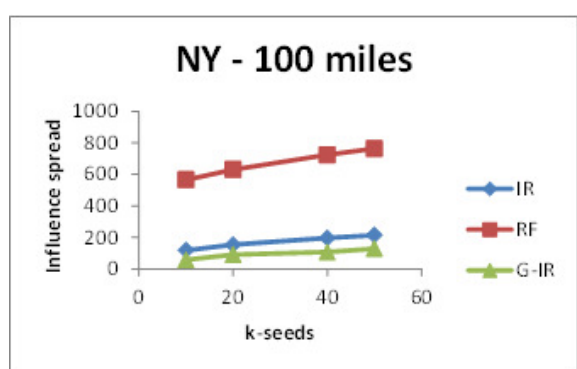

(a)

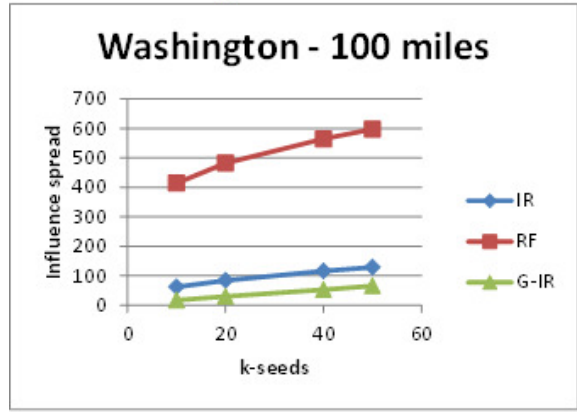

(c)

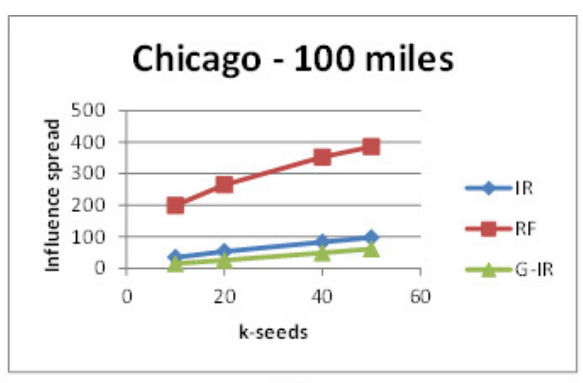

(b)

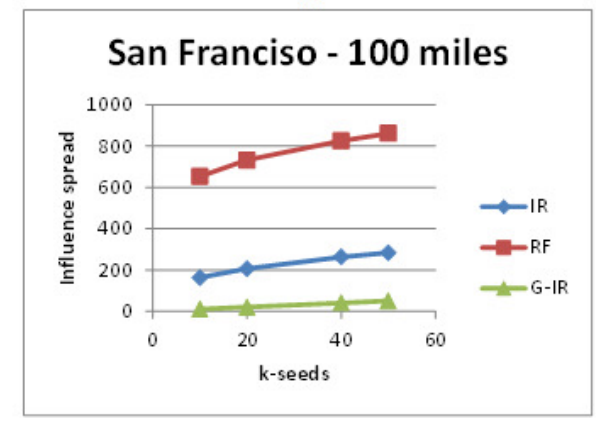

(d)

Figure 3. Influence spread on various regions on Gwalla dataset.

Figure 4 shows the results of influence spread of big cities of U.S. on Foursquare dataset. Since we do not have a considerable number of users and location check-in information of the Foursquare dataset, so it looks like that there is not much significant difference in influence spread achieved by all these algorithms. But we can observe the influence spread difference once the provided social network is large and consists of a vast amount of locations with check-in information.

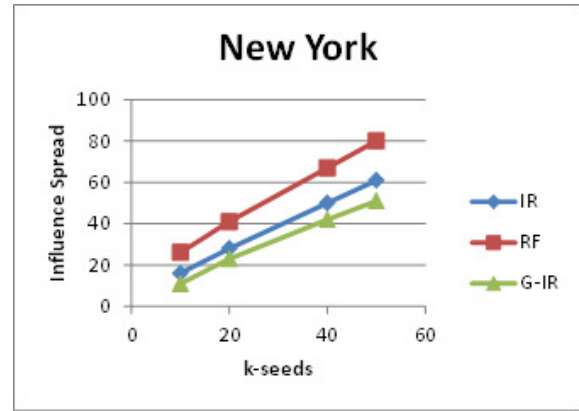

(a)

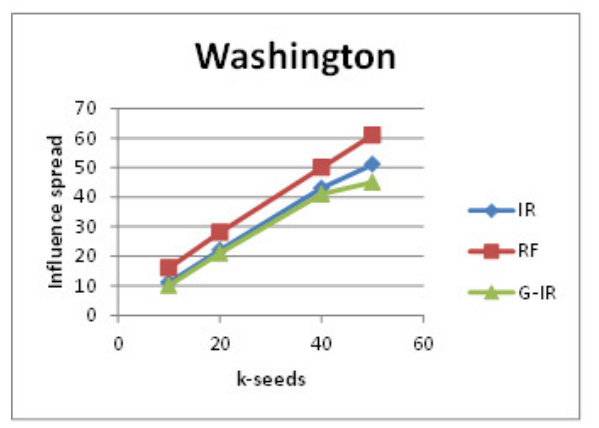

(c)

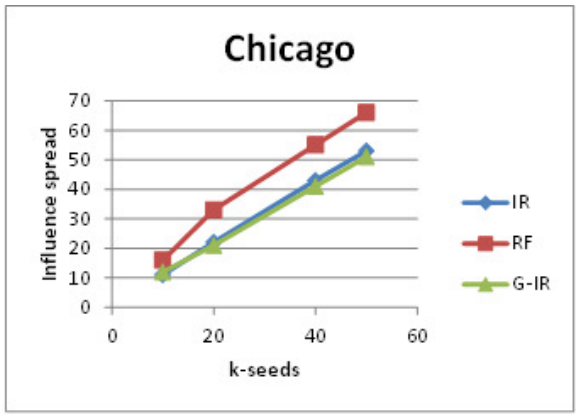

(b)

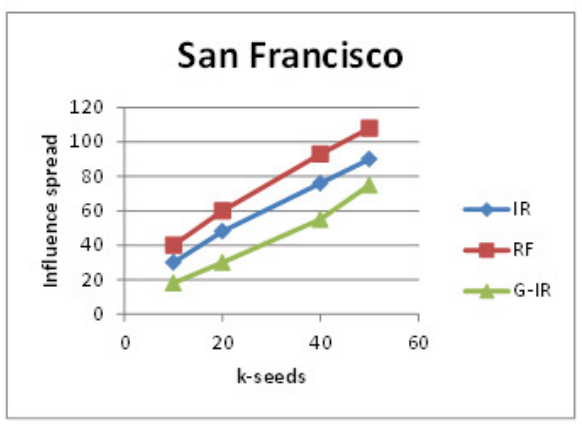

(d)

Figure 4. Influence spread on various regions on Foursquare dataset. 
It is evident from Figures 3 and 4 that the RF Selection algorithm achieved better results than IR Selection and G-IR Selection algorithms. IR and G-IR algorithms achieved similar influence spread in most of the cities on Foursquare datasets except in some cities where IR-algorithm performed better than G-IR.

From these experimental results, we believe that the top k-influential seed set who are local to the target city and have friends across the region can influence users across the target city and motivate them to visit famous locations under target cities.

Next, we discuss the effectiveness of a joint selection of influential users and locations selected by IR, RF, and G-IR algorithms.

Figure 5 shows the top- $k(k=20)$ influential users selected by G-IR, IR and FR algorithms respectively. It is observed that each algorithm produce a different $k$-influential seed set, so it is better to select the seed set which could maximize the product promotion. Further, we discuss the influential locations selected by each algorithm.

[148698, 130377, 145008, 145007, 104417, 148699, 68677, 56539, 83397, 19574, 41, 5524, 11275, 53473, $109503,4487,19216,137957,29984,63837]$

\section{G-IR Algorithm}

[148698, 38416, 145007, 13368, 106255, 18542, $33916,25275,114993,12604,11103,56539$, $11275,34050,107022,88597,541,3102,3105$, 191011]

\section{IR Algorithm}

[8091, 41, 34496, 467, 373, 5524, 499, 25275, 4487, 6598, 12604, 7301, 57007, 33839, 3978, $23616,11103,3855,4150,13080]$

\section{RF Algorithm}

Figure 5. Top-20 influential Users across San Francisco on Gwalla dataset.

Figure 6 shows the top-m $(m=20)$, influential locations selected by IR and FR selection algorithms with varying k-seeds. Figure 7 shows the same number of locations selected by the G-IR selection algorithm. It can be observed that when we increase the number of k-seeds, our proposed approach achieves better and similar influential locations as selected by a greedy approach with the highest users. However, the greedy approach (G-IR), which considers only check-in frequency distribution, yields poor results in influential locations selection. Locations selected by G-IR highlighted in red color shows the venues with less number of user visiting frequency than heuristic-based approaches.

Further, we conducted the same experiment for selecting influential locations on Foursquare and Gwalla datasets as well. Figures 8 and 9 show the results obtained by RF and GR-Selection algorithms on Foursquare dataset. Locations selected by G-IR highlighted in red color shows the venues with less number of users visiting frequency than our approach. 


\begin{tabular}{|cc||c|}
\hline loc_Id & \#users \\
23261.0 & 1048 \\
12505.0 & 923 \\
14128.0 & 888 \\
11844.0 & 819 \\
24963.0 & 746 \\
16399.0 & 689 \\
13022.0 & 453 \\
349982.0 & 443 \\
623366.0 & 416 \\
11875.0 & 350 \\
12313.0 & 335 \\
14151.0 & 302 \\
11794.0 & 296 \\
11834.0 & 257 \\
17417.0 & 236 \\
106840.0 & 232 \\
16907.0 & 228 \\
14148.0 & 214 \\
130586.0 & 187 \\
17710.0 & 179
\end{tabular} \mid \begin{tabular}{ccc}
23261.0 & 1048 \\
12505.0 & 923 \\
14128.0 & 888 \\
11844.0 & 819 \\
24963.0 & 746 \\
16399.0 & 689 \\
13022.0 & 453 \\
349982.0 & 443 \\
623366.0 & 416 \\
11875.0 & 350 \\
12313.0 & 335 \\
14151.0 & 302 \\
11834.0 & 257 \\
17417.0 & 236 \\
16907.0 & 228 \\
14148.0 & 214 \\
130586.0 & 187 \\
11797.0 & 153 \\
60450.0 & 152 \\
16310.0 & 145 \\
\hline
\end{tabular}

(a) $K=10, I R / F R$

\begin{tabular}{|c|c|c|c|}
\hline loc_ld & \#users & loc_id & \#users \\
\hline 23261.0 & 1048 & 23261.0 & 1048 \\
\hline 12505.0 & 923 & 12505.0 & 923 \\
\hline 14128.0 & 888 & 14128.0 & 888 \\
\hline 11844.0 & 819 & 11844.0 & 819 \\
\hline 24963.0 & 746 & 24963.0 & 746 \\
\hline 16399.0 & 689 & 16399.0 & 689 \\
\hline 12535.0 & 635 & 12535.0 & 635 \\
\hline 13022.0 & 453 & 13022.0 & 453 \\
\hline 349982.0 & 0 443 & 349982.0 & 443 \\
\hline 623366.0 & 0416 & 623366.0 & 416 \\
\hline 11875.0 & 350 & 11875.0 & 350 \\
\hline 12164.0 & 345 & 12164.0 & 345 \\
\hline 12313.0 & 335 & 12313.0 & 335 \\
\hline 12525.0 & 332 & 12525.0 & 332 \\
\hline 14151.0 & 302 & 14151.0 & 302 \\
\hline 11794.0 & 296 & 11794.0 & 296 \\
\hline 11834.0 & 257 & 11834.0 & 257 \\
\hline 12171.0 & 244 & 12171.0 & 244 \\
\hline 17417.0 & 236 & 17417.0 & 236 \\
\hline 12174.0 & 236 & 12174.0 & 236 \\
\hline
\end{tabular}

(b) $K=20, I R / F R$

\begin{tabular}{|cc|c}
\hline \multicolumn{2}{|c|}{ loc_id } & \#users \\
23261.0 & 1048 \\
12505.0 & 923 \\
14128.0 & 888 \\
11844.0 & 819 \\
24963.0 & 746 \\
16399.0 & 689 \\
12535.0 & 635 \\
24767.0 & 627 \\
13022.0 & 453 \\
349982.0 & 443 \\
623366.0 & 416 \\
11875.0 & 350 \\
12164.0 & 345 \\
12313.0 & 335 \\
12525.0 & 332 \\
14151.0 & 302 \\
11794.0 & 296 \\
11834.0 & 257 \\
12171.0 & 244 \\
11720.0 & 239
\end{tabular} \mid \begin{tabular}{ccc}
\multicolumn{2}{l|}{ loc_id } \\
23261.0 & \#users \\
12505.0 & 923 \\
14128.0 & 888 \\
11844.0 & 819 \\
24963.0 & 746 \\
16399.0 & 689 \\
12535.0 & 635 \\
24767.0 & 627 \\
13022.0 & 453 \\
349982.0 & 443 \\
623366.0 & 416 \\
11875.0 & 350 \\
12164.0 & 345 \\
12313.0 & 335 \\
12525.0 & 332 \\
14151.0 & 302 \\
11794.0 & 296 \\
11834.0 & 257 \\
12171.0 & 244 \\
11720.0 & 239 \\
\hline
\end{tabular}

(c) $K=40$, IR / FR

Figure 6. Top-20 influential locations selected by IR and RF algorithms across New York.

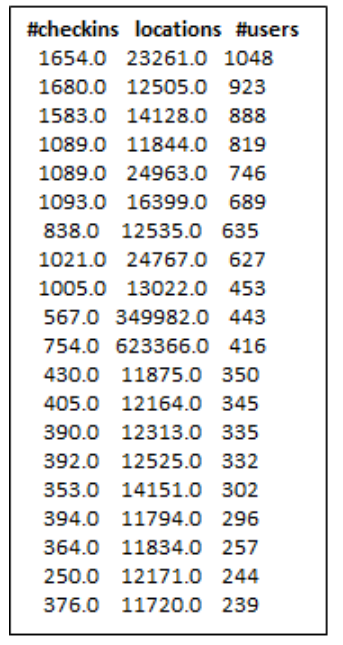

With highest user frequency

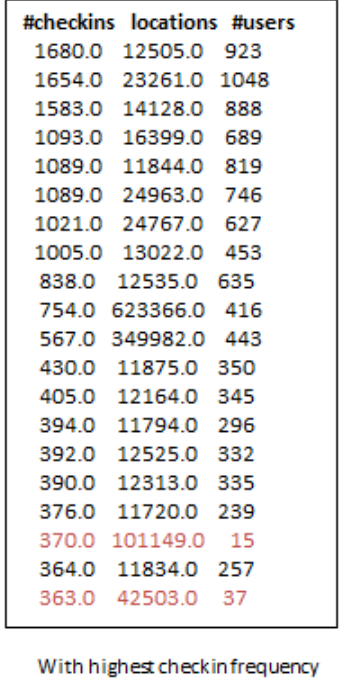

Figure 7. Top-20 influential locations across New York using G-IR algorithm.

\begin{tabular}{|c|}
\hline \#users \\
\hline 41059b00f964a520850b1fe3 682 \\
\hline 4bd2177d046076b055357371 409 \\
\hline 49d01698f964a520fd5a1fe3 161 \\
\hline 4a71e4cff964a520ccd91fe3 133 \\
\hline 43067280f964a52023271fe3 119 \\
\hline 49fdea19f964a5204a6f1fe3 80 \\
\hline $40 \mathrm{bbc} 700 \mathrm{f} 964 \mathrm{a} 520 \mathrm{~b} 1001 \mathrm{fe} 3 \quad 73$ \\
\hline $409 d 7480 f 964 a 520 f 2 f 21 e e 3 \quad 73$ \\
\hline $414 \mathrm{e} 1 \mathrm{~d} 80 \mathrm{f} 964 \mathrm{a} 520 \mathrm{ed} 1 \mathrm{c} 1 \mathrm{fe} 3 \quad 64$ \\
\hline $40 \mathrm{~b} 7 \mathrm{~d} 280 \mathrm{f} 964 \mathrm{a} 52093001 \mathrm{fe} 3 \quad 61$ \\
\hline 40f71a80f964a520afOa1fe3 60 \\
\hline $4390 a 026 f 964 a 5204 d 2 b 1 f e 358$ \\
\hline 49 ca8f4df964a520b9581fe3 55 \\
\hline $49 d 68 c 61 f 964 a 520$ e65c1fe3 54 \\
\hline $4468 f 484 f 964 a 5204 f 331 f e 3 \quad 53$ \\
\hline $44646408 f 964 a 52026331 \mathrm{fe} 3 \quad 53$ \\
\hline 3fd66200f964a520aDec1ee3 51 \\
\hline 42814b00f964a52002221fe3 50 \\
\hline 4aa48566f964a520024720e3 45 \\
\hline 42 bf4180f964a520b1251fe3 44 \\
\hline
\end{tabular}

$\mathrm{K}=10$

\begin{tabular}{|c|}
\hline \#users \\
\hline 41059b00f964a520850b1fe3 682 \\
\hline $4 \mathrm{bd} 2177 \mathrm{~d} 046076 \mathrm{~b} 055357371 \quad 409$ \\
\hline 49d01698f964a520fd5a1fe3 161 \\
\hline $4 a 71$ e4cff964a520ccd91fe3 133 \\
\hline $43067280 f 964 a 52023271 \mathrm{fe} 3119$ \\
\hline 42cc7080f964a520e9251fe3 104 \\
\hline 40c3b000f964a520e7001fe3 82 \\
\hline 49fdea19f964a5204a6f1fe3 80 \\
\hline 40bbc700f964a520b1001fe3 73 \\
\hline $409 \mathrm{~d} 7480 \mathrm{fg} 64 \mathrm{a} 52 \mathrm{fof} 2 \mathrm{f} 21 \mathrm{ee} 3 \quad 73$ \\
\hline 414e1d80f964a520ed1c1fe3 64 \\
\hline 40b7d280f964a52093001fe3 61 \\
\hline 40f71a80f964a520afOa1fe3 60 \\
\hline $4390 a 026 f 964 a 5204 d 2 b 1 f e 3 \quad 58$ \\
\hline 49ca8f4df964a520b9581fe3 55 \\
\hline $49 d 68 c 61 f 964 a 520$ e65c1fe3 54 \\
\hline $40 \mathrm{e} 4 \mathrm{a} 580 \mathrm{fg} 64 \mathrm{a} 52 \mathrm{fof} 5091 \mathrm{fe} 353$ \\
\hline $4468 f 484 f 964 a 5204 f 331 f e 3 \quad 53$ \\
\hline $44646408 f 964 a 52026331 \mathrm{fe} 3 \quad 53$ \\
\hline 3fd66200f964a520adec1ee3 51 \\
\hline
\end{tabular}

loc id \#users

41059b00f964a520850b1fe3 682 4 bd2177d046076b055357371 409 4a5824e5f964a5206db71fe3 210 4ab595e1f964a520877520e3 167 49d01698f964a520fd5a1fe3 161 4a71e4cff964a520ccd91fe3 133 49cc413df964a5205a591fe3 123 43067280f964a52023271fe3 119 42cc7080f964a520e9251fe3 104 40c3booof 964 a 520 e $7001 \mathrm{fe} 3 \quad 82$ 49fdea19f964a5204a6f $1 \mathrm{fe} 3 \quad 80$ 40943a00f964a520e5f21ee3 78 $409 \mathrm{~d} 7480 \mathrm{f} 964 \mathrm{a} 52 \mathrm{Of} 2 \mathrm{f} 21 \mathrm{ee} 3 \quad 73$ 40bbc700f964a520b1001fe3 73 4249 ec00f964a5208f201fe3 73 4bb9dbc 353649 c 74364 e48fb 72 444df333f964a5208d321fe 72 454 cf014f964a520c53c1fe3 65 $414 \mathrm{e} 1 \mathrm{~d} 80 \mathrm{f} 964 \mathrm{a} 520 \mathrm{ed} 1 \mathrm{c} 1 \mathrm{fe} 3 \quad 64$ 43728d80f964a520102a1fe3 63 $K=40$

Figure 8. Top-20 influential locations across San Francisco using RF-algorithm on Foursquare. 


\begin{tabular}{|c|c|}
\hline \#checkins & \#users \\
\hline 171341059 boofg64a520850b1fe3 & 682 \\
\hline 919 4bd2177d046076b055357371 & 1409 \\
\hline 364 4a5824e5f964a5206db71fe3 & 210 \\
\hline 323 4ab595e1f964a520877520e3 & 167 \\
\hline 440 49d01698f964a520fd5a1fe3 & 161 \\
\hline $3334 \mathrm{a} 71 \mathrm{e} 4 \mathrm{cff} 964 \mathrm{a} 520 \mathrm{ccd} 91 \mathrm{fe} 3$ & 133 \\
\hline $17149 \mathrm{cc} 413 \mathrm{df} 964 \mathrm{a} 5205 \mathrm{a} 591 \mathrm{fe} 3$ & 123 \\
\hline $16943067280 \mathrm{fg} 64 \mathrm{a} 52023271 \mathrm{fe} 3$ & 119 \\
\hline $12842 \mathrm{cc} 7080 \mathrm{f} 964 \mathrm{a} 520 \mathrm{eg} 251 \mathrm{fe} 3$ & 104 \\
\hline $10140 c 3 b 000 f 964 a 520$ e $7001 \mathrm{fe} 3$ & 82 \\
\hline 106 409ad180f964a520eef21ee3 & 82 \\
\hline $12349 \mathrm{fdea} 19 \mathrm{fg} 64 \mathrm{a} 5204 \mathrm{a} 6 \mathrm{f} 1 \mathrm{fe} 3$ & 80 \\
\hline $10040943 a 00 f 964 a 520 e 5 f 21$ ee3 & 78 \\
\hline 108 452b81ddf964a520393b1fe3 & 74 \\
\hline $82409 d 7480 f 964 a 520 f 2 f 21 \mathrm{ee} 3$ & 73 \\
\hline $1024249 e c 00 f 964 a 5208 f 201 f e 3$ & 73 \\
\hline $8940 \mathrm{bbc} 700 \mathrm{f} 964 \mathrm{a} 520 \mathrm{~b} 1001 \mathrm{fe} 3$ & 73 \\
\hline 93 4bb9dbc353649c74364e $48 \mathrm{fb}$ & 72 \\
\hline $107444 \mathrm{df} 333 \mathrm{f} 964 \mathrm{a} 5208 \mathrm{~d} 321 \mathrm{fe} 3$ & 72 \\
\hline $893 \mathrm{fd} 66200 \mathrm{f} 964 \mathrm{a} 52041$ ee1ee3 & 70 \\
\hline
\end{tabular}

With highest user frequency

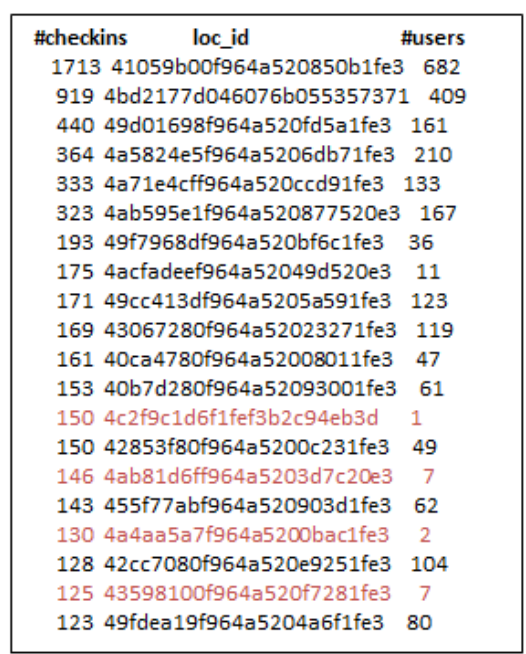

With highest checkin frequency

Figure 9. Top-20 influential locations across San Francisco using GIR algorithm.

\subsection{2. t-IUL Approaches Result Summary}

We conducted the experiments for $t$-IUL problem on Foursquare dataset only because it have location category information while Gowolla and Brighkite does not have any location category information. We selected the target region, $R$, as Log Angeles and category topic, $q$, as food. We have considered "food" as a location category type as users are found to share favorite food locations with their friends. Here, we discuss the influential locations selected by t-RF and t-GIR algorithms and see the results difference produced by both algorithms. Influential seeds, k, selected by t-RF and t-GIR algorithms are almost the same for the Foursquare dataset, so we have not shown that result in this paper.

Figure 10 shows the top- 10 influential locations selected by the $\mathrm{t}-\mathrm{RF}$ algorithm. We found around $5.3 \mathrm{k}$ check-ins matching query category and target city. Besides, we set k-seeds as 50 because there are not many users in Foursquare dataset. Influential users visit the 95 unique venues. Further, Figures 11 and 12 show the venues selected by $t$-GIR Selection algorithm considering highest users with check-in frequency. The venues highlighted in red color shows the drawback of $t$-GIR algorithm because it returns locations with the highest check-ins, but only a few users have visited those places

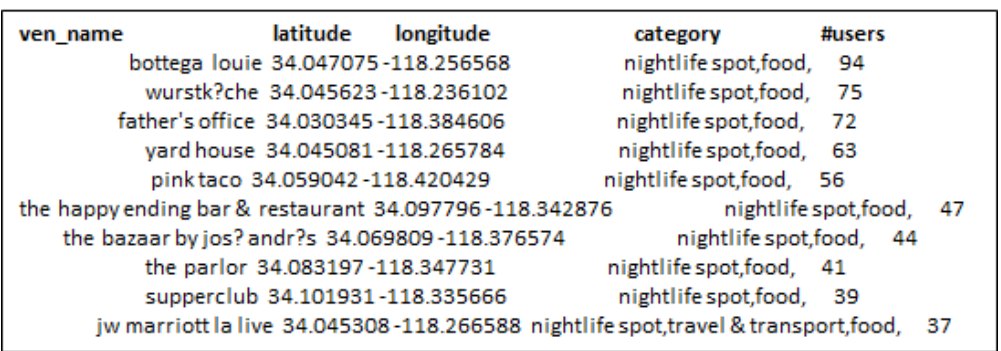

Figure 10. Top-10 influential locations in Log Angles using t-RF algorithm.

\begin{tabular}{|c|c|c|c|}
\hline latitude longitude & \#users \# & \#checkin & \\
\hline bottega louie $34.047075-118.256568$ & nightlife spot,food, 94 & 20 & \\
\hline wurstk?che $34.045623-118.236102$ & nightlife spot,food, 75 & 88 & \\
\hline father's office $34.030345-118.384606$ & nightlife spot,food, 72 & & \\
\hline yard house $34.045081-118.265784$ & nightlife spot,food, 63 & & \\
\hline pink taco $34.059042-118.420429$ & nightlife spot,food, 56 & & \\
\hline $\begin{array}{l}\text { el rey theatre } 34.062698-118.348880 \text { music ve } \\
\text { canter's delicatessen } 34.078780-118.361716\end{array}$ & $\begin{array}{l}\text { enue, food,arts \& entertainment, } \\
\text { nightlife spot,food. } 53\end{array}$ & $\begin{array}{c}55 \\
69\end{array}$ & 70 \\
\hline the happyending bar \& restaurant $34.097796-118.34287$ & 76 nightlife spot,food, & 47 & 63 \\
\hline $\begin{array}{l}\text { kogi bbq truck } 34.045832-118.256836 \\
\text { the bazaar by jos? andr?s } 34.069809-118.376574\end{array}$ & $\begin{array}{c}\text { travel \& transport,food, } 47 \\
\text { nightlife spot,food, } 44\end{array}$ & 56 & \\
\hline
\end{tabular}

Figure 11. Top-10 influential locations in Log Angles using t-GIR algorithm. 


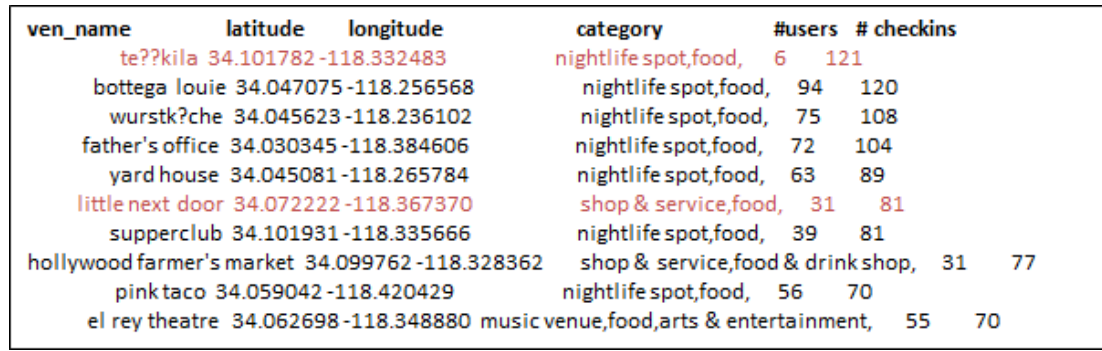

Figure 12. Top-10 influential locations in Log Angles using t-GIR algorithm considering check-ins.

\subsection{Discussion}

The experimental results provides the following analysis.

- There is a significant percentage difference in influence spread within and out of the target region.

- $\quad$ RF-algorithms achieved better performance as they considered users having connections across the target city for promotion. The top k-influential seed set who are local to the target city and have friends across the region can influence users to visit famous locations under target city.

- $\quad$ Third, the locations selected by considering check-in information only are not always an optimal choice for production promotion. Since, few users can have checked-in location hundreds or thousands times but it may not denote its real popularity.

- When we increase the number of k-seeds, our proposed approach achieves better influential locations as selected by a greedy approach with the highest users. However, the greedy approach (G-IR), which considers only check-in frequency distribution, yields poor results in influential locations selection.

\section{Conclusions}

In this paper, we have studied the problem of finding influential users and locations jointly at the target query region for product promotion. Further, we studied the selection of influential users and locations matching the specific location category. As location popularity values, such as the number of check-ins, are not always available, so we have proposed heuristic-based methods that can find influential users and locations in the target region. Experimental results showed that our heuristic-based methods achieved better performance than the greedy-based methods. Further, our proposed algorithms can easily be extended by adding more constraints such as recommending top $M$ locations, by considering the distance between each location, i.e., we can suggest influential locations for product promotion which should be distant apart from each other. In future work, we would like to find a co-relation between $k$-influential users and $m$-influential locations under target region in location-based social networks.

Author Contributions: K.A. and C.-T.L. formulated the problem and proposed a methodology. C.-T.L. provided an insightful analysis about results and guided in the proposed methodology. K.A. implemented the proposed method and prepared the initial paper draft. Y.-S.C. contributed in manuscript draft and provided suggestions in results analysis. All authors have read and agreed to the published version of the manuscript.

Funding: This work is supported by Ministry of Science and Technology(MOST) of Taiwan under grants 109-2636-E-006-017 (MOST Young Scholar Fellowship) and 109-2221-E-006-173, and also by Academia Sinica under grant AS-TP-107-M05.

Institutional Review Board Statement: Not applicable.

Informed Consent Statement: Not applicable.

Data Availability Statement: The data presented in this study are available on request from the corresponding author.

Conflicts of Interest: The authors declare no conflict of interest. 


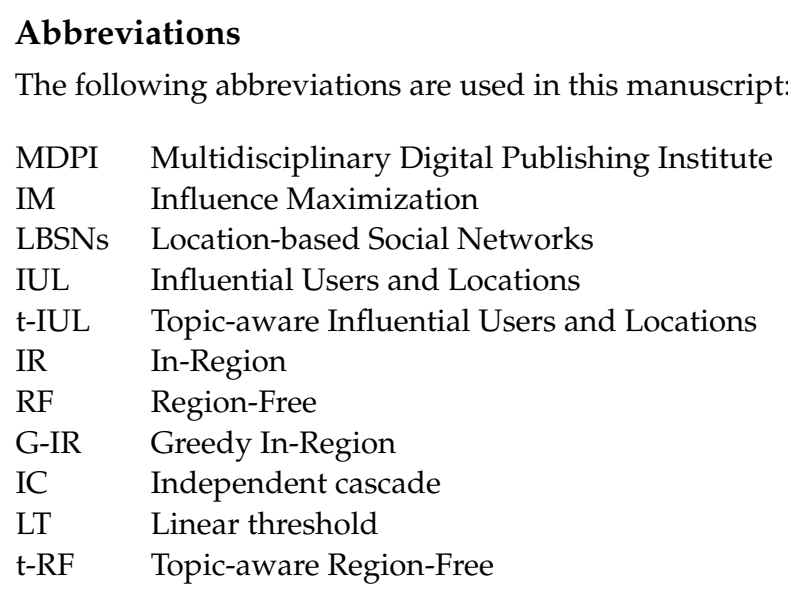

\section{References}

1. Kempe, D.; Kleinberg, J.; Tardos, É. Maximizing the spread of influence through a social network. In Proceedings of the Ninth ACM SIGKDD International Conference on Knowledge Discovery and Data Mining, Washington, DC, USA, 24-27 August 2003; pp. 137-146.

2. Chen, W.; Wang, Y.; Yang, S. Efficient influence maximization in social networks. In Proceedings of the 15th ACM SIGKDD International Conference on Knowledge Discovery and Data Mining, Paris, France, 28 June-1 July 2009; pp. 199-208.

3. Chen, W.; Wang, C.; Wang, Y. Scalable influence maximization for prevalent viral marketing in large-scale social networks. In Proceedings of the 16th ACM SIGKDD International Conference on Knowledge Discovery and Data Mining, Washington, DC, USA, 24-28 July 2010; pp. 1029-1038.

4. Borgs, C.; Brautbar, M.; Chayes, J.; Lucier, B. Maximizing social influence in nearly optimal time. In Proceedings of the Twenty-Fifth Annual ACM-SIAM Symposium on Discrete Algorithms, Portland, OR, USA, 5-7 January $2014 ;$ pp. 946-957.

5. Ohsaka, N.; Akiba, T.; Yoshida, Y.; Kawarabayashi, K.I. Fast and Accurate Influence Maximization on Large Networks with Pruned Monte-Carlo Simulations. In Proceedings of the AAAI Conference on Artificial Intelligence, Quebec City, QC, Canada, 27-31 July 2014; pp. 138-144.

6. Tang, Y.; Shi, Y.; Xiao, X. Influence maximization in near-linear time: A martingale approach. In Proceedings of the 2015 ACM SIGMOD International Conference on Management of Data, Melbourne, Australia, 31 May-4 June 2015; pp. $1539-1554$.

7. Domingos, P.; Richardson, M. Mining the network value of customers. In Proceedings of the Seventh ACM SIGKDD International Conference on Knowledge Discovery and Data Mining, San Francisco, CA, USA, 26-29 August 2001; pp. 57-66.

8. Richardson, M.; Domingos, P. Mining knowledge-sharing sites for viral marketing. In Proceedings of the 8th ACM SIGKDD International Conference on Knowledge Discovery and Data Mining, Edmonton, AB, Canada, 23-26 July 2002; pp. 61-70.

9. Gao, H.; Liu, H. Data analysis on location-based social networks. In Mobile Social Networking; Springer: Berlin/Heidelberg, Germany, 2014; pp. 165-194.

10. Li, N.; Chen, G. Analysis of a location-based social network. In Proceedings of the 2009 International Conference on Computational Science and Engineering, Vancouver, BC, Canada, 29-31 August 2009; Volume 4, pp. 263-270.

11. Li, G.; Chen, S.; Feng, J.; Tan, K.L.; Li, W.S. Efficient location-aware influence maximization. In Proceedings of the 2014 ACM SIGMOD International Conference on Management of Data, Snowbird, UT, USA, 22-27 June 2014; pp. 87-98.

12. Bouros, P.; Sacharidis, D.; Bikakis, N. Regionally influential users in location-aware social networks. In Proceedings of the 22nd ACM SIGSPATIAL International Conference on Advances in Geographic Information Systems, Dallas/Fort Worth, TX, USA, 4-7 November 2014; pp. 501-504.

13. Zhu, W.Y.; Peng, W.C.; Chen, L.J.; Zheng, K.; Zhou, X. Modeling user mobility for location promotion in location-based social networks. In Proceedings of the 21th ACM SIGKDD International Conference on Knowledge Discovery and Data Mining, Sydney, NSW, Australia, 10-13 August 2015; pp. 1573-1582.

14. Wang, X.; Zhang, Y.; Zhang, W.; Lin, X. Distance-aware influence maximization in geo-social network. In Proceedings of the 32nd International Conference on Data Engineering, Helsinki, Finland, 16-20 May 2016.

15. Leskovec, J.; Krause, A.; Guestrin, C.; Faloutsos, C.; VanBriesen, J.; Glance, N. Cost-effective outbreak detection in networks. In Proceedings of the 13th ACM SIGKDD International Conference on Knowledge Discovery and Data Mining, San Jose, CA, USA, 12-15 August 2007; pp. 420-429.

16. Ahmad, T.; Li, X.J.; Seet, B.C.; Cano, J.C. Social Network Analysis Based Localization Technique with Clustered Closeness Centrality for 3D Wireless Sensor Networks. Electronics 2020, 9, 738. [CrossRef]

17. Yuan, Q.; Cong, G.; Sun, A. Graph-based point-of-interest recommendation with geographical and temporal influences. In Proceedings of the 23rd ACM International Conference on Conference on Information and Knowledge Management, Shanghai, China, 3-7 November 2014; pp. 659-668.

18. Levandoski, J.J.; Sarwat, M.; Eldawy, A.; Mokbel, M.F. Lars: A location-aware recommender system. In Proceedings of the 2012 IEEE 28th International Conference on Data Engineering, Washington, DC, USA, 1-5 April 2012; pp. 450-461. 
19. Berjani, B.; Strufe, T. A recommendation system for spots in location-based online social networks. In Proceedings of the 4th Workshop on Social Network Systems, Salzburg, Austria, 10 April 2011; p. 4.

20. Zheng, V.W.; Zheng, Y.; Xie, X.; Yang, Q. Collaborative location and activity recommendations with GPS history data. In Proceedings of the 19th International Conference on World Wide Web, Raleigh, NC, USA, 26-30 April 2010; pp. 1029-1038.

21. Ye, M.; Yin, P.; Lee, W.C. Location recommendation for location-based social networks. In Proceedings of the 18th SIGSPATIAL International Conference on Advances in Geographic Information Systems, San Jose, CA, USA, 2-5 November 2010 ; pp. 458-461.

22. Gao, H.; Tang, J.; Hu, X.; Liu, H. Content-Aware Point of Interest Recommendation on Location-Based Social Networks. In Proceedings of the AAAI Conference on Artificial Intelligence, Austin, TX, USA, 25-30 January 2015; pp. $1721-1727$.

23. Zhang, J.D.; Chow, C.Y. Point-of-interest recommendations in location-based social networks. SIGSPATIAL Spec. 2016, 7, 26-33. [CrossRef]

24. Leskovec, J.; Krevl, A. SNAP Datasets: Stanford Large Network Dataset Collection. 2014. p. 49. Available online: http: / / snap.stanford.edu/data (accessed on 10 December 2020).

25. Wang, W.; Yin, H.; Chen, L.; Sun, Y.; Sadiq, S.; Zhou, X. Geo-SAGE: A geographical sparse additive generative model for spatial item recommendation. In Proceedings of the 21th ACM SIGKDD International Conference on Knowledge Discovery and Data Mining; ACM: New York, NY, USA, 2015; pp. 1255-1264. 